\title{
ROBOTIC PROCESS AUTOMATION - A VIRTUAL ASSISTANT FOR INVENTORY MANAGEMENT
}

\author{
SINDHU PRASANTHI DASU ${ }^{1} \&$ CH. RADHAKUMARI ${ }^{2}$ \\ ${ }^{I}$ Department of Management \& Commerce, Sri Sathya Sai Institute of Higher Learning, \\ Anantapur Campus, Anantapur, India \\ ${ }^{2}$ Professor, Department of Management and Commerce, Sri Sathya Sai Institute of Higher Learning,
}

Anantapur Campus, Anantapur, India

\begin{abstract}
Robotic process automation (RPA) is an emerging form of business process automation technology based on the notion of software robots or Artificial Intelligence (AI) workers. The term RPA can be split as Robotic representing an entity which is capable of being programmed by a computer for doing computer tasks, known as robot; Process signifying the sequence of actions taken in a logical order to achieve a particular end; and Automation suggesting the accomplishment of tasks without any human intervention. In RPA, bots are software programs which automate the routine tasks, and mimic human behavior with no change to the existing infrastructure. Intelligent Process Automation (IPA) enhances the cognitive abilities of software bots by bringing intelligence to them and paves the path for the software robots to mimic the way in which human brain works. In this context a research is undertaken to develop a virtual text to speech assistant bot that aids a manager who has the responsibility of managing inventory of items besides his varied other jobs. The customized software robot ensures that if the stock in the inventory of any item reaches reorder level, bots itself sends the message for re-ordering the necessary items through an email to the supplier. The study demonstrates that RPA can be a better substitute to a human being which can perform time intense and necessary activities more effectually.

KEYWORDS: Intelligent Process Automation, Robotic Process Automation, Cognitive Skills \& Virtual
\end{abstract}

Received: Nov 11, 2018; Accepted: Dec 01, 2018; Published: Dec 15, 2018; Paper Id.: IJRRDJUN20191

\section{INTRODUCTION}

Robotic Process Automation (RPA) is defined by the Institute for Robotic Process Automation as 'the application of technology allowing employees in a company to configure computer software or a 'robot' to capture and interpret existing applications for processing a transaction, manipulating data, triggering responses and communicating with other digital systems.' The Institute also states that the companies who employ extensive work force for outrageous amount of knowledge centered process flow will be profited from RPA as it will enhance their competencies and save time and money. So, RPA mimics human behavior to execute an action without human intervention with the help of software robots. 'Bots' are the software programs that imitate the behavior of a human which automate routine tasks and eliminate inefficiency. The integration of cognitive abilities to Robotic Process Automation has led to the emergence of new field known as Intelligent Process Automation which paved way for the bots to aid managers in decision making. With a managerial use case, an attempt is made in the current research paper todevelop a text to speech assistant bot to an inventory manager of a small enterprise using UiPath software which will notify him with the current available inventory level and places order for the items to be ordered by triggering an email to the supplier. 


\section{REVIEW OF LITERATURE}

The review of literature for the current paper is presented in three sections. The first section deals with the inventory management system of the Small Businesses and the second section deals with the text to speech technology, the third part deals with the areas where RPA can be applied. Inventorymanagement is one of the most important functions of a business. The objective of an inventory management is to design policies that will achieve optimal inventory investment (Kontuš, 2014). The author explored the relation between companies' inventory level and profitability with respect to return on asset. The researcher also developed a model for figuring the net savings from modifications made in the inventory level. Inventory management can largely impactan organization's performance and it is much more important and complex in case of SME (Ferenčíková, 2014). The author identified the main problems in the inventory management system of manufacturing companies as flexibility and efficiency. The researcher concluded that the factors causing these problems have significant influence on time, quality and cost.Small and medium scale enterprises are in many cases confronted with the challenge of inadequate inventory of raw materials which lead to underutilization of their capacity and thus hindered the business growth (Monisola, 2013). The author examined various inventory management practices of SMEs and discovered that there is an extensive gap between theoretic and practical implementation of good practices.

A virtual assistant is a software agent that can execute tasks or services for an individual(Takawale, et al., 2017). The authors described distinct types of virtual assistants; the domains to which these assistants can be developed viz. medical, healthcare and education. In addition, the authors also discussed the challenges encountered in applying these assistants. Virtual assistants are capable enoughto back users in fetching the right information (Claessen, et al., 2017). The author assessed the information service quality rendered by three virtual assistants on e-commerce websites as well as analyzed user perception of those assistants. Text to speech synthesis is a rapidly emergent facet of computer technology. It has played a major role in the way humans interact with system (Isewon, et al., 2014). The author developed a text to speech synthesizer that reads aloud a large volume of text for visually impaired people. Text to speech implementation is useful in industries such as banking, railways and as an aid to physically challenged persons, language education (Poonam.S.Shetake, et al., 2014). The authors surveyed approaches that are used for text to speech conversion and concluded that creation of data base, optical character recognition are basis for text to speech conversion.

RPA is that technology that helps the organization's to have a competitive edge and is geared up to transform the logistics industry (Bhaskar, 2017). The author mentioned the use cases where RPA can help logistics automate process of repetitive nature namely billing in trucking companies, tracking of shipments and automating inventory management. He also stated about the importance of combining RPA with Artificial Intelligence (AI), which is known as 'Cognitive Automation' so that predictions and support decision-making was facilitated. The author referred to a study conducted by Global tech research firm's Info Services Group in 2017 which states that by $2019,72 \%$ of companies use RPA to minimize cost, reduce transaction time, increase productivity in addition to improvement in levels of compliance. He concluded that RPA is preferred by enterprises as it proposes immediate, low cost approach to automate basic, rule based processes without reengineering them and there is no trade-off between quality and efficiency.

The applications of RPA range from Healthcare, HR, banking to Finance and many more.(RPA at Work:Focus on Manufacturing, 2018) discusses the application of RPA in the field of Finance and Accounting. This case talk about the automation of a routine process called "Global Finance Audit Delivery "in "Global Professional Services \& Audit Company', which was a client of UiPath. This recurring process of auditing the financial reports had several fill in 
processes, which included cross-footing, re-calculation of balance sheets etc. The case describes that UiPath has provided solution to this challenge by standardizing the business process logic and automating forty-five percentage of the audit process, which resulted in saving 54,000 hours of work annually.

(RPA at Work:Focus on Health Care, 2018) confers about the successful implementation of RPA in a leading US healthcare service provider. The case describes about the automation of the manual process, which involved exporting daily reports from an application by deploying a robot using UiPath. The case further states that the automation had resulted in elimination of human error and attaining an accuracy rate of $100 \%$ in processing.

(RPA at Work:Focus on Health Care, 2018) explains the effective implementation of RPA by UiPath in a global manufacturing company for automating the process of vendor management. The case also describes the challenges faced by the company namely high cost of operation and propensity to errors. It discusses the solution provided by UiPath, which was automating the entire vendor management process of creating a vendor, VAT validation for European and Non-European countries by deploying a robot. This automation brought out an increased efficiency, reduction in manual errors leading to improvement in operational performance.

Although a lot has been said about RPA at the enterprise level many companies are still in the post-experimentation phase while others are still discovering how projects perform implementing it(Deckard, 2018). The author also mentions that there is a need for good product support, easy access to training and right partnership to progresses towards more AI. The author substantiates the above argument by discussing the research conducted by Everest Group, which surveyed 72 enterprises and found security, scalability and software richness to be the most important characteristics. In the light of the current requirement in the inventory management system of small industries, the current research is undertaken to find a reliable solution using RPA.

\section{SIGNIFICANCE OF STUDY}

In today's global, dynamic and challenging economy no business is either too small or too big to confront the competitionand sustain its competitive advantage. However, there is an emergent need for small scale enterprises to be more rapid and agile in their processes so as to survive in the market.Most of the small scale enterprises start with elementary infrastructure which may incorporate a blend of basic accounting software and document-centred processes like spreadsheets etc. Acquiring an enterprise level infrastructure for managing business processes is a prerequisite yet a huge investment for small business. In a small company, employees sport multiple caps and raise up to the occasion only when the need exits. As the business expands, it lands up in fragmented disorganised corporate structure comprising of numerous programs that handle finance, inventory, sales etc. Although, Enterprise Resource Planning (ERP) solutions are provided to small businesses as it involves considerable expenses and the companies' budget is limited, in-depth evaluation and selection of the vendor that offers high return on investment is essential. Moreover, these small enterprises do not have the technical support to deploy these ERP solutions. Hence business processes became more mundane and are prone to errors as voluminous amount of data had to be processed from workflow to workflow.

Inventory management is one such critical business process that has to be managed efficiently. Underestimation of raw material of an inventory often results in improper orders effecting customer satisfaction. On the other hand, upholding excess inventory level leads to depletion of raw material. The cost of inefficient inventory management is both excessive and unnecessary. If the management controls the trade-off between the holding cost of inventory and cost of 
overstocking efficiently, the capital saved can be diverted to improve other areas like production, salesetc. The aim of an inventory management system is to convey the manager 'When to order?'\&'How much to order?'. To fulfill this aim, a need for a virtual speech to text assistant arose which would assist the inventory manager of small enterprise. According to a survey conducted by Spiceworks, a social network site in April 2018, it is found that $40 \%$ of large businesses across North America and Europe expected to deploy AI voice assistants in their workplace by 2019.The study also concluded that $29 \%$ of organizations have deployed one or more AI assistants for professional tasks, or planned to deploy one or more in the next one year. In this context, the present study is significant as it is a novel attempt as a virtual text to speech and an email assistant for inventory management system of a small enterprise is developed.

\section{OBJECTIVES OF THE STUDY}

The current research aims at demonstrating the potential of a RPA robot as a virtual text to speech and an email assistantfor inventory management through a managerial use case. The study also highlightshowa busy manager of the current corporate world can employ RPA as an effective and reliable assistant, to perform the repetitive human tasks more competently and efficiently. With the 'bot' taking over the task of a human assistant to ensure that if the stock in the inventory of a small enterprise is at the required level and automatically reorder the necessary items through an email to the supplier, the study intends at demonstratingthat RPA can be a better substitute to a human being as an assistant to a manager which can perform time intense, unproductive nevertheless necessary activitiesmore effectually.

\section{METHODOLOGY FOR ACHIEVING THE OBJECTIVES}

The study adopted the following methodology to achieve the objective set. In RPA two types of bots, namely an attended bot and an unattended bot are available. An attended bot performs its automation on the user's local workstation and needs a human to trigger or schedule them. These are called Front Office Robots (FOR). An unattended 'bot' that runs on a virtual machine and executes automation that does not require user interaction. They can be triggered from a server. They are called as Back Office Robots (BOR). The following description deals with the development of RPA methodology using FOR.

RPA Development Methodology: The stages to automate the inventory management process via FORs isdescribed below:

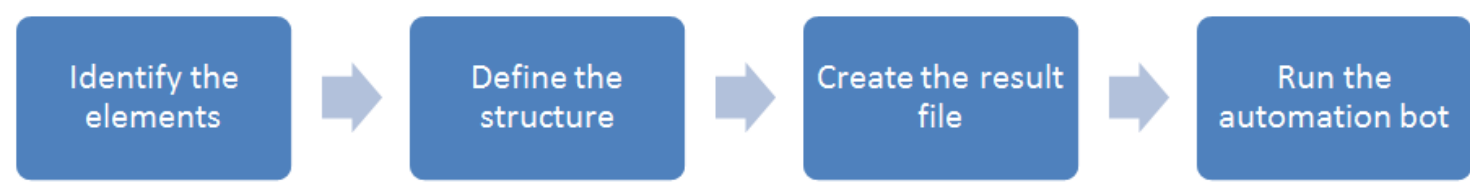

Any RPA development project should follow a structured stage-wise procedure. A typical APA development has the following four phases.
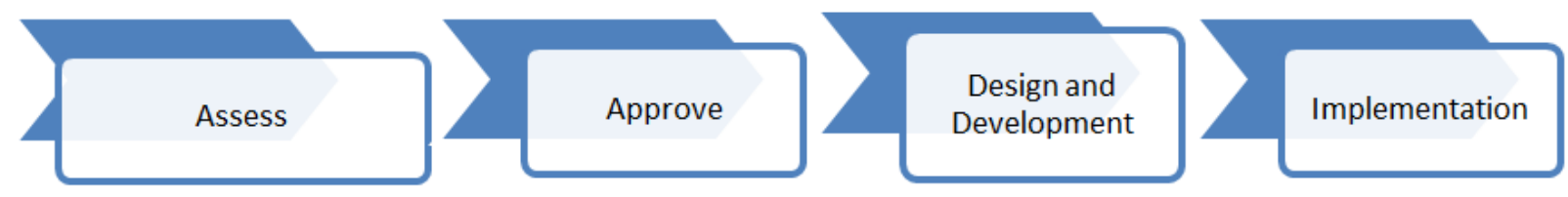

Asses phase begins with the investigation of the process that could be automated. Stage concludes by drawing the feasibility of the RPA project in more detail. Approve phase starts with the approval of the process, which is to be 
automated. It is followed by a detailed investigation and documentation of the process. After documentation, the future robotic process will be designed. The stage generally ends with a business case being presented to the management team. Design phases commences by looking at which software best fulfils the criteria outlined in the business case. During this phase, the robot will be designed with the exact process. With agile iterations, the robot will be programmed to scale the level of automation. The stage ends with the testing of the robot. In the implementation phase, the robot is deployed into a working environment, mimicking the behavior of an employee.

The current research project carried out recognizes the real time application of RPA in managerial administration and is depicted using UiPath community edition software. UiPath is a leading RPA tool, is user friendly, and has a free community edition. It provides a complete solution for application integration, automating applications, administrative and business IT tasks and processes. A manager working in an inventory of a small businesshas daily chore of attending to multiple jobsranging from the procurement of the raw materials to their delivery to the respective destinations. A simulated managerial use case is considered in which all the items of the inventory are classified into three categories namely 'A','B' and 'C'as mentioned by selective inventory control or ABC analysis. A voice assisted software bot is scheduled to run through the excel sheet containing the class of items and notifies the manager about the no. ofeach class of items namely class ' $\mathrm{A}$ ', 'B' and ' $\mathrm{C}$ ' are on hand in the inventory. The bot also verifies inventory levels and initiates re-orders for quantitieswhich fall belowthe minimum setreorder level for each class of itemby automatically updating the excel sheet with the information of items to be reordered andtriggers a mail to the supplier.The creation of an automation project requires the following steps:

\section{Step 1: Open UiPath Community Edition Software as Shown Below}

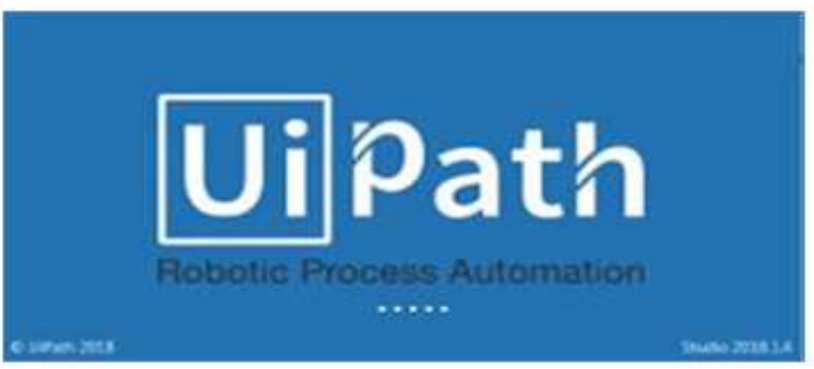

\section{Step 2: Create Blank Project}

A window will appear asking for project name as shown below. Provide a name and to the project and the description of the project and click on create, a project will be created, as shown below. 


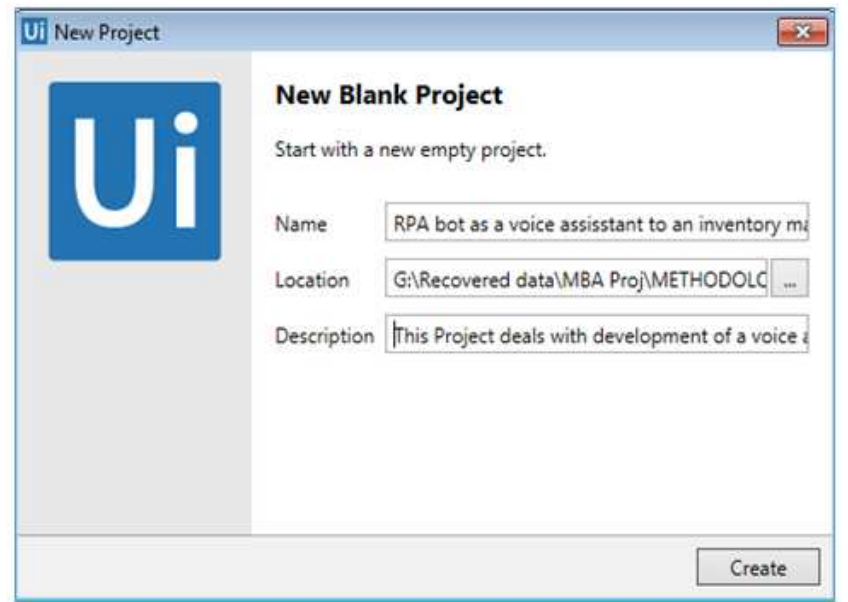

\section{Step 3: Selection of New Sequence}

On the design tab, in the File Group, select New Sequence. The New Sequence Diagram window is displayed as shown below. Create Main workflow as depicted below.

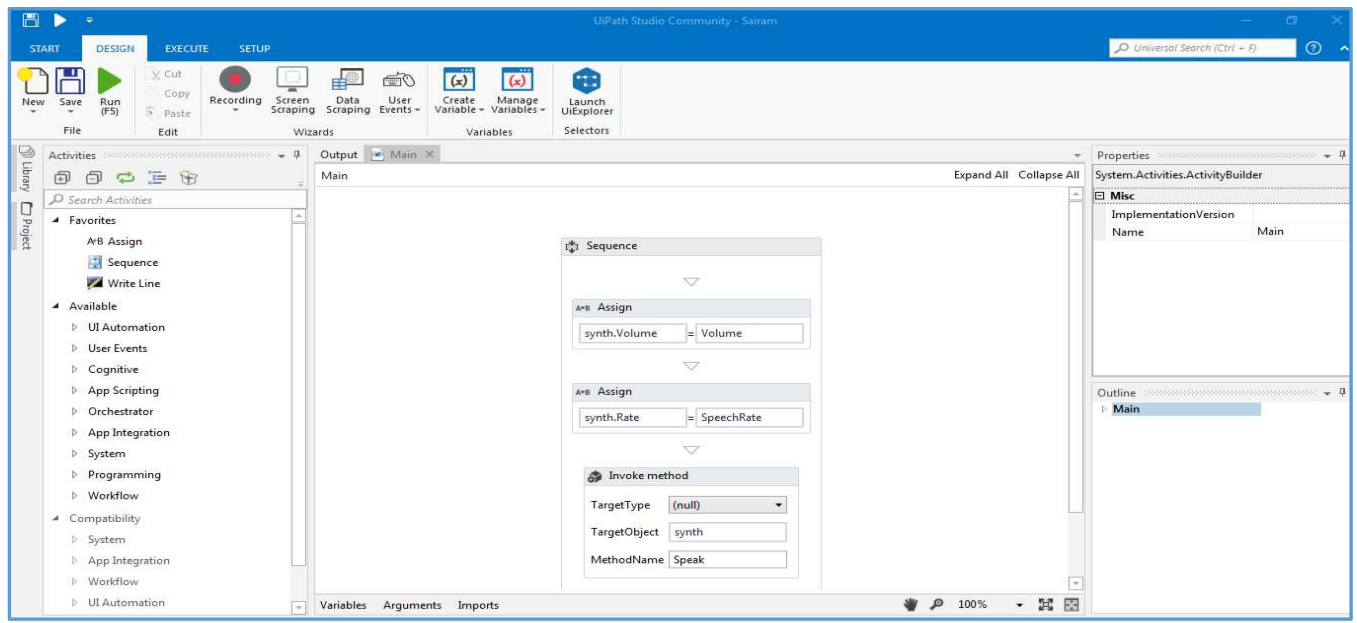

\section{Step 4: Declaration of Variables}

The initialization of 'Speech Synthesizer' variable is the primary activity for a voice bot. This variable can be invoked from the activity with property name as UiPath. System. Synthesis. Speech Synthesizer. The variables which are created for the main file are declared as shown below

\begin{tabular}{|l|l|l|l|}
\hline Name & Variable type & Scope & Default \\
\hline synth & SpeechSynthesizer & Sequence & new SpeechSynthesizer \\
\hline work & Workbook & Sequence & Enter a VB expression \\
\hline wo & WorkbookApplicatio Sequence & Enter a VB expression \\
\hline Create Variable & & & \\
\hline & & & \\
\hline
\end{tabular}

\section{Step 5: Creation of the 'Read from File' Sequence}


On the design tab, in the File Group, select New Sequence. The New Sequence Diagram window is displayed. Create a new sequence and name it as 'Read from file. 'Declare a variable named 'steps' which would appeal to the text to speech functionality of the software. Invoke the main workflow which is created in step 3 as shown below.

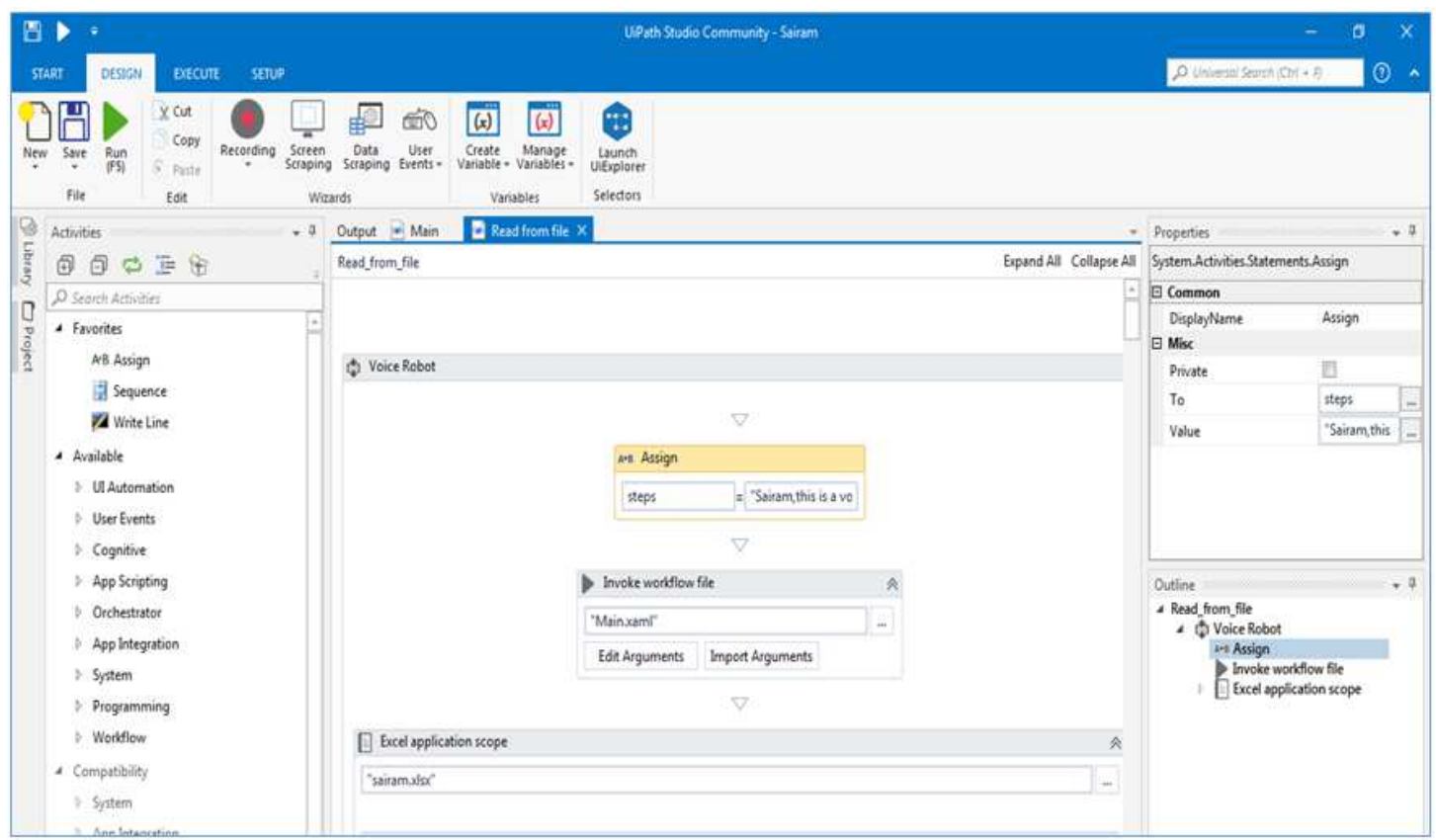

\section{Step 6: Importing the Arguments from the Workflow}

Create the arguments in the main file as shown below which will be further imported by the 'Read from file' workflow.

\begin{tabular}{|l|l|l|l|l||}
\hline Name & Direction & Argument type & Default value \\
\hline Volume & In & Int32 & 100 \\
\hline SpeechRate & In & Int32 & -2 \\
\hline Text & In & String & "Sairam" & \\
\hline Create Argument & & & & \\
\hline & & & & \\
\hline
\end{tabular}

\section{Step 7: Creation of 'Read Range Activity'}

In the excel application scope, add read range activity and specify the sheet name which is to be read by the bot as 'Sheet 1' as shown below. Also, add an 'assign' activity wherein the variable 'steps' is assigned to the particular text that is to be converted to speech. Createa for each loop using 'for each' activity so that each row of the sheet is considered as shown below. 


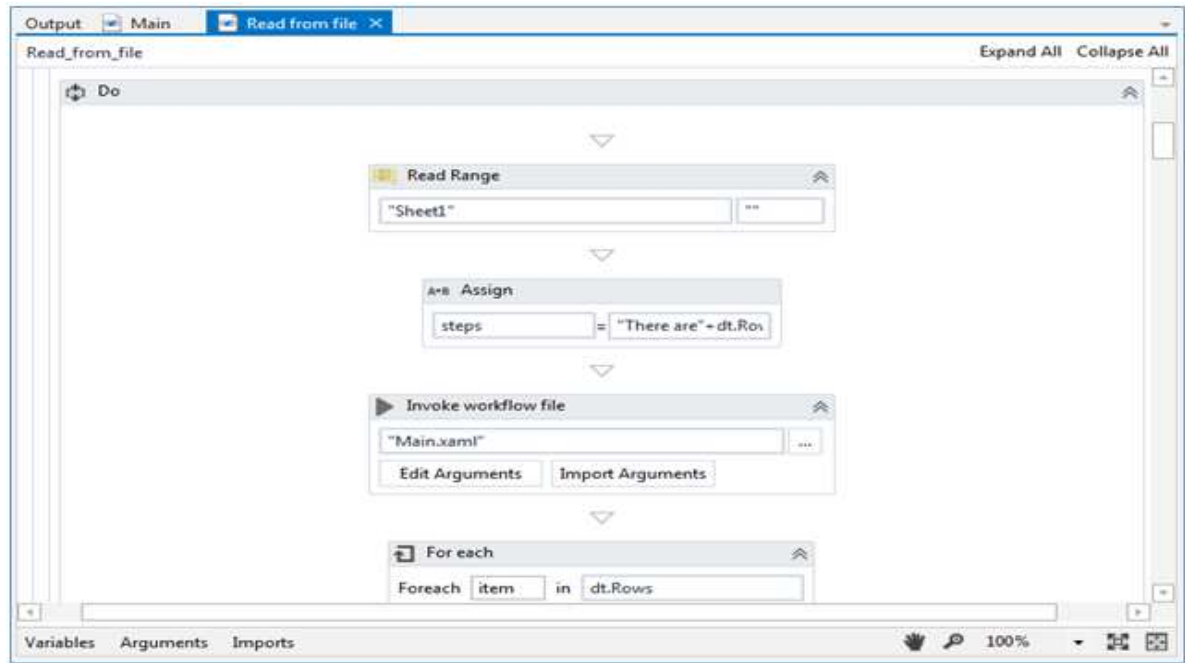

\section{Step 8: Creation of 'if' Condition for the Specific Target Reorder Level}

In the body of the loop create an 'if "condition to compare the inventory level with the set reorder level for each row as shown below. Declare the variable 'status' and assign the variable to 'reorder' if the 'if' condition is satisfied. Assign the variable 'steps' to the 'status' value to bring voice robot into action as shown below.

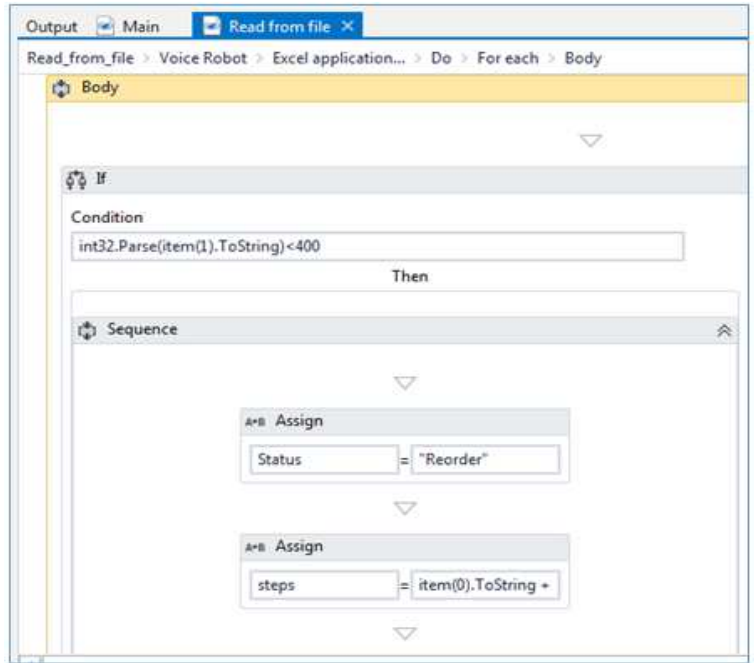

\section{Step 9: Inclusion of 'for Each Row' Activity Inside the 'for Each Loop'}

Inside the for each loop after the if condition, add a for each row activity as shown below.

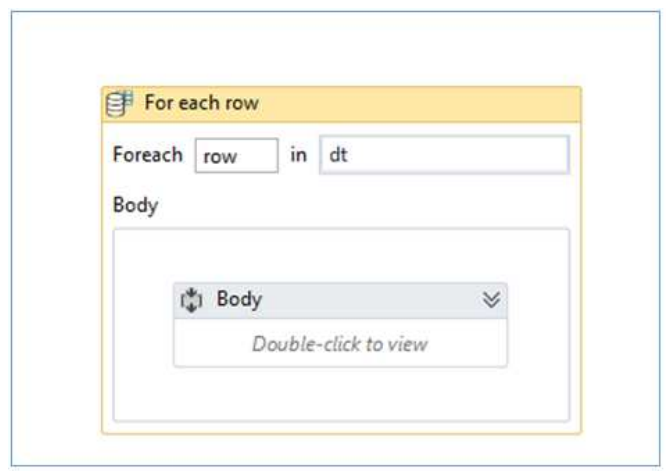




\section{Step 10: Creation of Excel Application Scope}

In the excel application scope, add 'write cell' activity and mention the name of the sheet as 'reorder' as shown below.

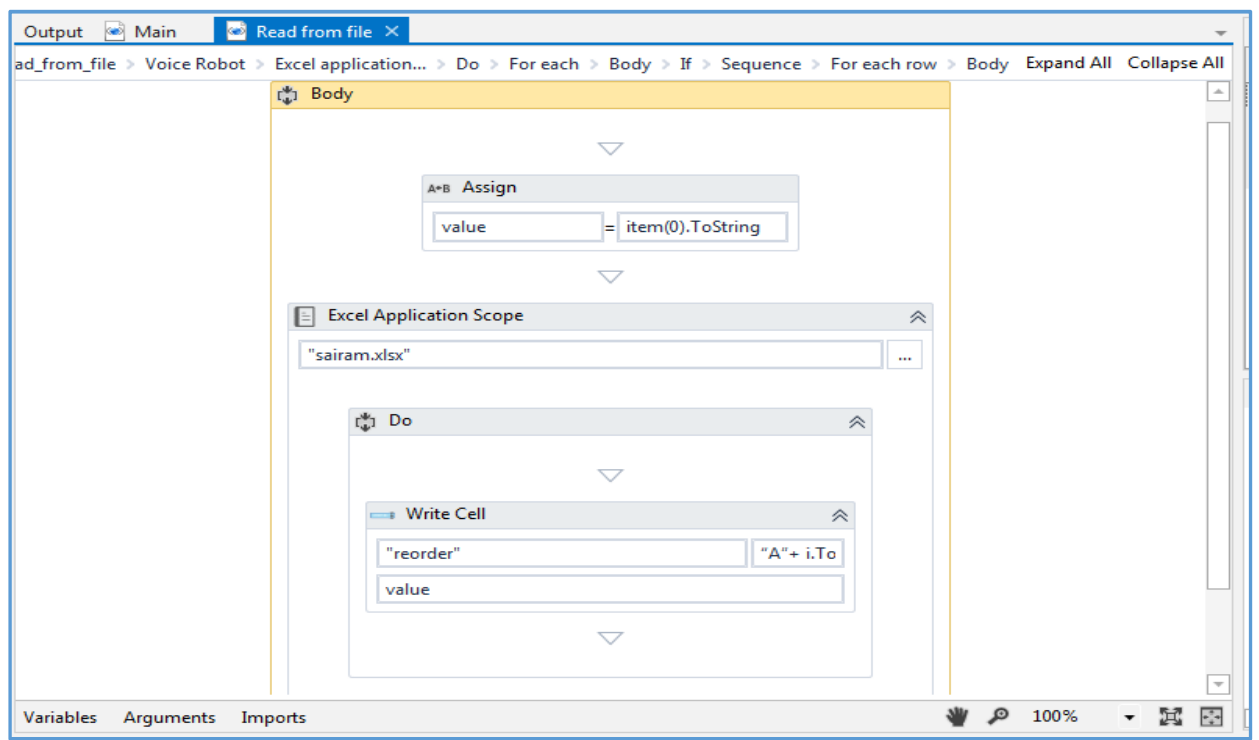

\section{Step 11: Incrementing of Variable}

Declare a variable 'i' which runs through every row and increment it as shown below.

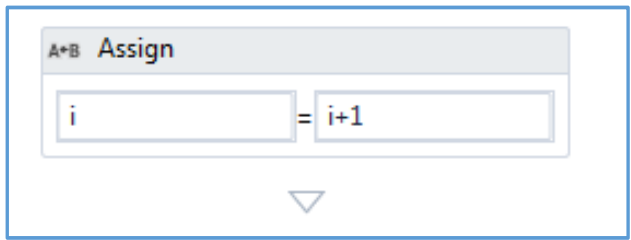

\section{Step 12: Creation of 'Else' Condition}

In the body of the loop, create an else condition and assign the 'status' to 'don't reorder' as shown below.

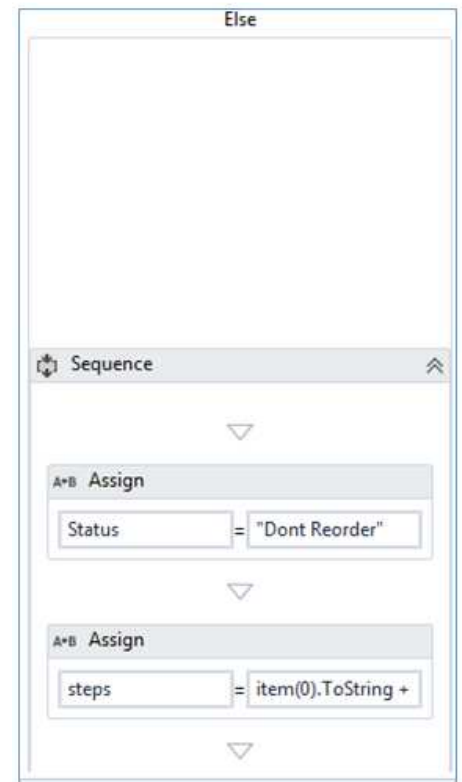




\section{Step 13: Invoking the Workflow File}

Invoke the workflow of main file using arguments and assign the 'steps' variable to the text that is to be spoken by voice bot as shown below

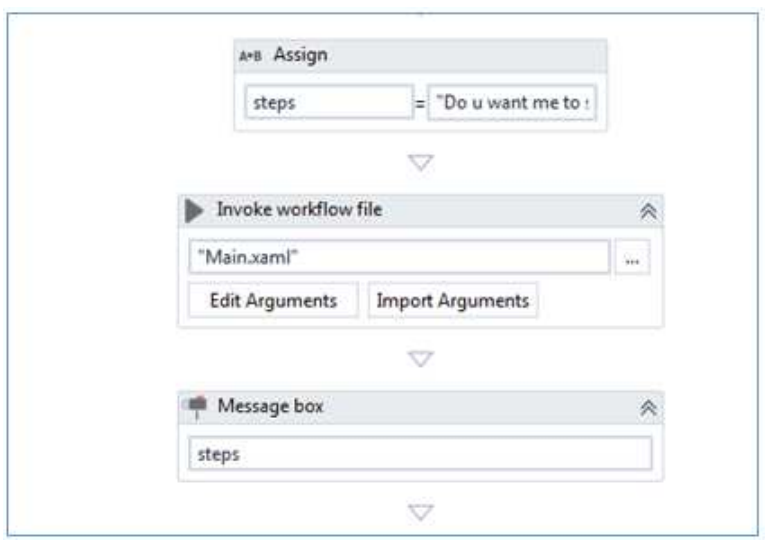

\section{Step 14: Creation of 'if' Condition}

Create an 'if' condition that triggers a message to manager for his approval to send mail to the supplier as shown below.

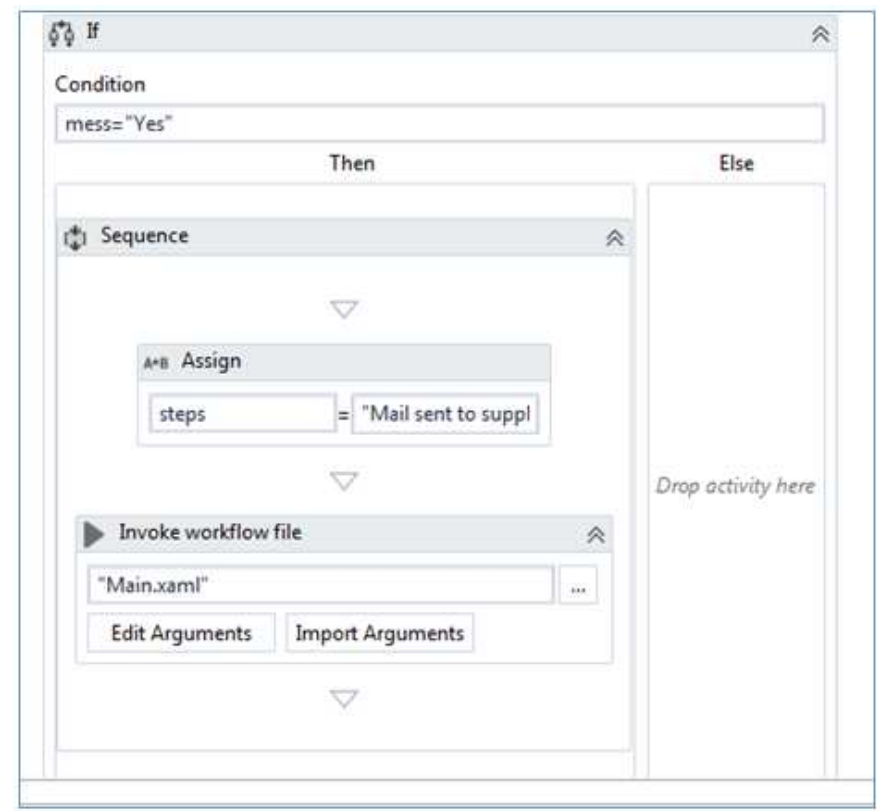

\section{Step 15: Creation of 'Outlook Mail Activity'}

Create an outlook mail activity with the subject and body of the mail as shown below. 


\begin{tabular}{|l|l|} 
To Send Outlook Mail Message \\
To "supplier.atp@gmail.com" \\
Subject "Reorder list of inventory" \\
Body "Hello Sir,Please reorder the following items" \\
\\
Attach Files
\end{tabular}

The above automated process of inventory management with the aid of bot can be scheduled with the help of UiPath Orchestrator where in there is no need for the bot to execute it. The UiPath orchestrator is a web application that enables the manager not only to deploy, schedulebots on an hourly, daily, weekly or even a monthly basis but also to monitor and manage the bots. As the orchestrator is a web application, the manager is expected to have net connectivity. The processes built in the UiPath Studio are deployed into the orchestrator and a schedule is created to run the bots so that there is no need for manager to waste his time on tedious jobs.

\section{OUTCOME OF THE RESEARCH}

The outcome of the research work undertaken encompasses the comparison between the process of inventory management before and after implementation of RPA. The first part depicts the process followed in the inventory management manually.The second part deals with the depiction of the process of automating the inventory management using RPA.

\section{BEFORE THE IMPLEMENTATION OF RPA}

The manual process of inventory management in small enterprise usually consists of the following stages. The stage begins with the procurement of raw materials followed by the monitoring and entering of the inventory transactions details into the system to prepare decisive information. When the no. of items in the inventory are abundant, the whole process of managing the inventory becomes tedious leading to a fair chance of errors in the operations and culminates the time of manager which could otherwise be used for vital activities.

\section{AFTER THE IMPLEMENTATION OF RPA}

Successful implementation of RPA equips the manager with a virtual text to speech assistant bot that runs through the details of the transactions and notifies the manager on the class of items available in the inventory, create a file of items to be re-ordered and triggers a mail to the supplier after his approval. Illustrated below are the figures that depict the inventory sheet with each class of items and re-order sheet updated by the bot. 


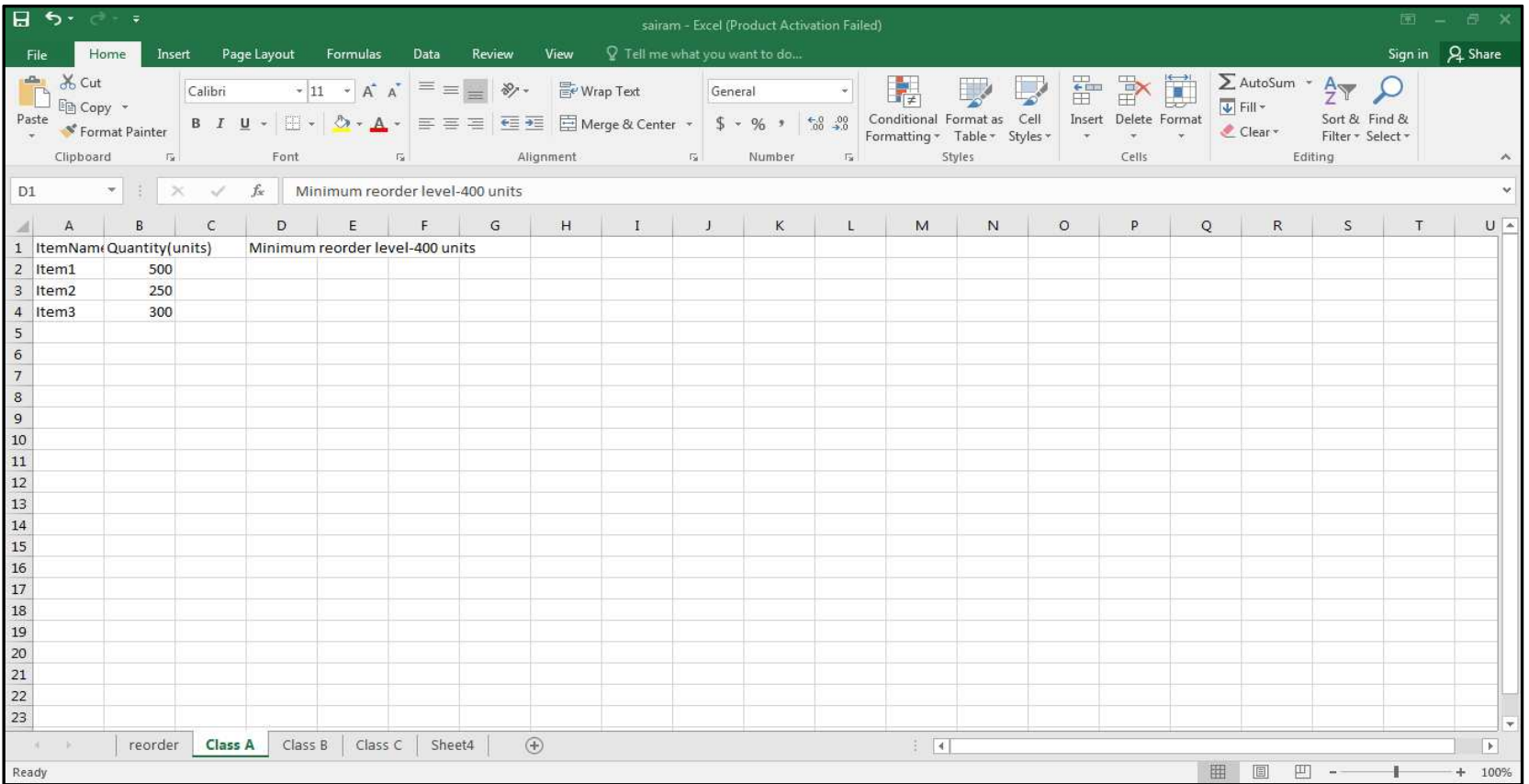

Figure 1: Depicting the Inventory Sheet with Each Class of Items Namely 'A','B' and 'C'

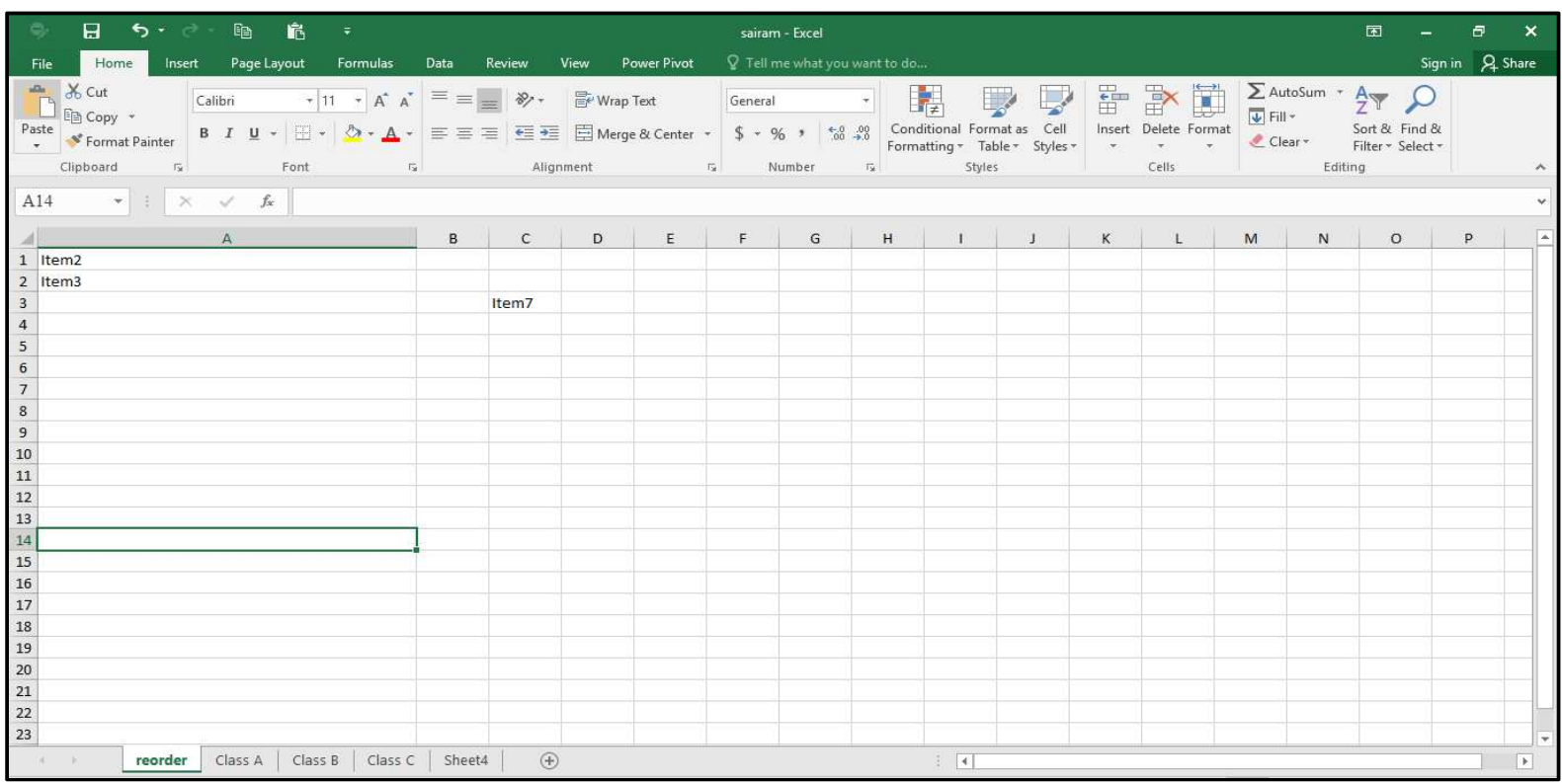

Figure 2: Depicting the Inventory Reorder Sheet Updated with Items to be Reordered

The following figure shows the message initiated by the bot to manager for approval. If clicked 'yes', the bot triggers a mail to the supplier.

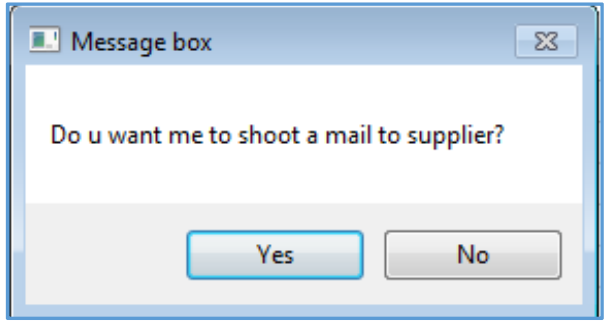

Figure 3: Depicting the Message Initiated by the Bot 


\section{COMPETITIVE ADVANTAGE OF THE APPLICATION}

In recent years although ERP systems and traditional IT systems gave a break to the inventory managers by supporting them in monitoring the re-order level of items, these technologies are huge investments and the resources of small enterprises are limited. As the managers of small businesses take on them the responsibilities of all levels, they don't get competent time for critical activities, disparities arising in managing operations result in increased costs and decreased productivity of the jobs. In this regard, RPA bot addresses this problem by working at operational level as a virtual text to speech and mail assistant. The benefit of this bot is that it aids the manager who multitasks by notifying him aloud of the no. of items available in the inventory and also by ordering the necessary items which supports the business to work efficiently and deliver effective results.

\section{CONCLUSIONS}

In the present study, the potential of RPA as a virtual text to speech assistant is unleashed through a managerial use case of inventory management system in a small business. It dealt on how a busy manager of a small firm in the corporate world can engage RPA as an assistant in the place of a human being for notifying him on the inventory available and aid him in operating more productively and constructively.

\section{REFERENCES}

1. Bhaskar, W., 2017. rpa-robot-process-automation-is-the-cheapest-investment-with-highest-returns-that-any-logisticscompany-should-do. [Online] Available at: http://dreamorbit.com [Accessed 16 July 2018].

2. Claessen, V., Schmidt, A. \& Heck, T., 2017. Virtual Assistants A Study on the Usability and User Perception. Berlin, Humboldt-Universitätzu Berlin, pp. 116-130.

3. Deckard, M., 2018. enterprise-rpa-2018-research-everest. [Online] Available at: https://www.uipath.com [Accessed 16 July 2018].

4. Ferenčíková, D., 2014. Inventory Management in Small and Medium-Sized Manufacturing. Bali, Proceedings of the 2014 International Conference on Industrial Engineering and Operations Management.

5. Kontuš, E., 2014. Management of inventory in a company. EkonomskiVjesnik, 27(2), pp. 245-256.

6. Monisola, O., 2013. An Assessment of Inventory Management in Small and Medium Scale Industrial Enterprises in Nigeria. European Journal of Business and Management, 5(28), pp. 150-158.

7. Poonam. S. Shetake, S. A. Patil \& Jadhav, P. M., 2014. Review of Text to Speech Conversion Methods. International Journal of Industrial Electronics and Electrical Engineering, 2(8), pp. 29-35.

8. RPA at Focus: Finance and Accounting (2018) UiPath.

9. RPA at Work: Focus on Health Care (2018) UiPath.

10. RPA at Work: Focus on Manufacturing (2018) UiPath.

11. Spiceworks, 2018. spiceworks-study-reveals-40-percent-large-businesses-will-implement-intelligent-assistants-chatbots-2019. [Online] Available at: https://www.spiceworks.com [Accessed 28 November 2018].

12. Takawale, N. et al., 2017. A Study on Virtual Assistant in Artificial Intelligence. International Journal of Innovative Research in Computer and Communication Engineering, 5(9), pp. 15142-15145 
